\title{
Impact of gravitational interaction between the Moon and the Earth on the occurrence of episodes of cardiogenic pulmonary edema in the field
}

\author{
ROMAN SKULEC ${ }^{1,2,3}$, JITKA CALLEROVA ${ }^{2}$, JIRI KNOR $^{2,4}$, PATRIK MERHAUT ${ }^{2}$, STEFAN SKULEC ${ }^{5}, K_{\text {KARL KUCERA }}^{2}$, VLADIMIR CERNY $^{1,6,7}$ \\ 1 Department of Anesthesiology, Perioperative Medicine and Intensive Care, J.E. Purkinje University, \\ Masaryk Hospital Usti nad Labem, Usti nad Labem, Czech Republic \\ 2 Emergency Medical Service of the Central Bohemian Region, Kladno, Czech Republic \\ 3 Department of Anesthesiology and Intensive Care, Charles University in Prague, Faculty of Medicine in \\ Hradec Kralove, University Hospital Hradec Kralove, Hradec Kralove, Czech Republic \\ 4 3rd Medical Faculty, Charles University in Prague, Prague, Czech Republic \\ 5 Slovak Hydrometeorological Institute, Bratislava, Slovak Republic \\ 6 Department of Anesthesia, Pain Management and Perioperative Medicine, Dalhousie University, Halifax, Nova Scotia, Canada \\ 7 Department of Research and Development and Department of Anesthesiology and Intensive Care, Charles University in \\ Prague, Faculty of Medicine in Hradec Kralove, University Hospital Hradec Kralove, Hradec Kralove, Czech Republic
}

Corresponding author

Roman Skulec

ZZSSCK

Prof. Veseleho 461, Beroun 266 01, Czech Republic

Phone: 00420777577497

E-mail:skulec@email.cz

\section{ABSTRACT}

While circadian variation of occurrence of cardiovascular emergencies has been described, it has not been assessed whether fluctuations of gravitational interaction between the Earth and the Moon may induce other types of its variation in time have the similar impact. Therefore, we decided to evaluate whether there is an association between the occurrence of prehospital cardiogenic pulmonary edema (CPE) episodes treated by Emergency Medical Services (EMS) and fluctuations in the intensity of gravitational interaction between the Earth and the Moon.

Methods. We extracted all dispatches to CPE episodes from the EMS database of the Central Bohemian Region, Czech Republic, between 2.11.2008 and 1.7.2014. For each episode, the intensity of gravitational interaction between the Moon and the Earth was calculated. The study period was divided into 11 sections of equal duration according to the different intensity of gravitational interaction, and occurrence of CPE was compared among the groups. Results. We observed up to 4,744 episodes of CPE during the study period. Occurrence of CPE episodes was highest in the periods with the weakest intensity of gravitational interaction $(\leq 1.80 \mathrm{e} 1026 \mathrm{~N})$, while in the periods of the most intense gravitational interaction $(\geq 2.26 \mathrm{e} 1026 \mathrm{~N})$, the lowest proportion of CPE cases was observed (23.44 vs. $3.79 \%, \mathrm{p}<0.001)$. Conclusions. We identified a significant association between the intensity of gravitational interaction between the Earth and the Moon and occurrence of CPE, treated by our EMS. The weakest intensity was associated with its increased occurrence and vice versa. Further research is required for potential use of this phenomenon in a chronotherapeutic approach to secondary prevention of CPE.

Key words: cardiogenic pulmonary edema, gravitational interaction

\section{INTRODUCTION}

Interaction of two celestial bodies, the Earth and the Moon, has long been the subject of mystical speculation but also of serious research. There have been many published reports of the impact of the Moon's phases on the occurrence of cardiovascular diseases, psychiatric disorders, rheumatologic disorders, birth rates, the risk of sudden cardiac death and the number of emergency department visits or hospital admissions. (1-11)

In the vast majority, a link was not identified. Even the shifts of lunar phases are often considered a marker of oscillation of the gravitational field of the Moon; in fact, there is no direct relationship between these natural phenomena. Moreover, rather than gravitational field of the Moon itself, we should consider the gravitational interaction between the Moon and the Earth. It has a significant impact on the hydrosphere, atmosphere and the Earth's core. Research on its potential effect on regular variation of occurrence of diseases in time may be important not only for a detailed understanding of its pathophysiology but it also can have practical implications for the operation of emergency medical systems and emergency departments and for a chronotherapeutic approach to the management of diseases. However, a very limited number of studies concerning this topic have been published. $(8,12)$

One of the frequent and well defined cardiovascular emergencies with welldefined circadian variation is cardiogenic pulmonary edema (CPE). Therefore, we selected CPE as a model disease to evaluate whether there is an association between the occurrence of CPE episodes and fluctuations in the intensity of the gravitational interaction between the Earth and the Moon in a defined region for a specified time period and if so, whether there is a difference between the patterns of interaction in clinical types of CPE. A secondary aim of the study was to assess whether there is an association between the occurrence of CPE episodes and moon phases. We hypothesized that there is no significant association between the occur- 
rence of CPE episodes and the intensity of the gravitational interaction between the Earth and the Moon.

\section{MATERIALS AND METHODS}

We performed a retrospective study, conducted in accordance with the Declaration of Helsinki. The Emergency Medical Service of the Central Bohemian Region is the exclusive provider of primary pre-hospital emergency care in the Central Bohemian Region, Czech Republic. The region includes both the rural and urban population, representing a total of 1315299 inhabitants on a total area of $11015 \mathrm{~km} 2$. A computer search of patients treated for dyspnea between 2.11.2008, 02:38 and 1.7.2014, 12:03 was conducted. Only those with CPE were entered in the study. Each patient was managed by a physician in the field. Criteria for considering the patient having CPE were leading complaint of shortness of breath, presence of bilateral pulmonary rales on the pre-hospital physical examination and the absence of another explanation for the clinical setting. The criteria were evaluated by two investigators independently. The results of their analysis were compared with the diagnosis established by the physician in the field. In the case of full accordance with the diagnosis of CPE, the patients were selected for analysis. In the case that both investigators and the physician in the field excluded pulmonary oedema, the patient was not included. If there was a partial mismatch, the case was carefully re-evaluated including personal consultation with the EMS physician and in-hospital documentation.

Patients with missing data were not included in the study. According to the first measured systolic blood pressure (SBP) in the field, we assigned the patients into three clinical subgroups for further analysis: CPE with hypertension (SBP $>140 \mathrm{~mm}$ $\mathrm{Hg}$ ), CPE with normotension (SBP $\geq 90$ and $\leq 140 \mathrm{~mm} \mathrm{Hg}$ ) and CPE with hypotension (SBP $<90 \mathrm{~mm} \mathrm{Hg}$ ). Initial blood pressure measurement was performed following the guidelines by oscillometric automatic sphygmomanometer validated according to standardized protocol and checked periodically through calibration. (13) In those patients with more than one initial blood pressure measurement available, the average value was calculated for further analysis.

The time of occurrence of the CPE episode was defined as the time the call was received by the dispatch centre and determined with an accuracy of 1 minute. While the distance from the centre of the Earth to the centre of the Moon regularly fluctuates, it was calculated for each CPE episode separately. Intensity of the gravitational interaction $(\mathrm{F})$ between the Moon and the Earth for each $\mathrm{CE}$ event was calculated using the formula (g gravitational constant; $\mathrm{m} 1$ weight of the earth, kg; m2 weight of the Moon, kg; r distance from the centre of the Moon to the centre of the Earth, m). The study period was divided into 11 sections of equal duration according to the different intensity of gravitational interaction, from weakest to strongest. Occurrence of CPE was compared among the subgroups.

We also compared the number of CPE episodes in the time points of apogee and perigee (the points in the orbit of the Moon at which it is farthest or nearest to the Earth, respectively) \pm 36 hours during the whole survey period. For that purpose, events from the time period 12.11.2008, 16:56 to 04.06.2014, 16:26 were used to maintain the same number of apogee and perigee ( 74 of each).

The exact date and time of moon phases in the reporting period were obtained from NASA sources. Each synodic month (the period of a complete revolution of the Moon around the Earth, as the period between successive new moons equal to 29.531 days) was divided into four equal periods lasting 7.383 days with a particular phase of the moon in the middle of the period. Each period has been named by the corresponding phase of the moon. For each of the four types of periods we calculated the number of CPE events and compared the differences among the groups.

\section{STATISTICAL ANALYSIS}

For the statistical analysis, mean values \pm $\mathrm{SD}$ or frequencies (percentages) were calculated as necessary. Differences between groups were compared using the $\chi^{2}$ test, and statistical significance was calculated by the Fischer exact test for alternative variables. Statistical significance for continuous variables was determined by the paired Student t-test. The collected occurrence numbers were compared using Poisson distribution and regression. Comparison of subgroups according to systolic blood pressure was performed by ANOVA analysis. Data were analyzed using Microsoft Excel 2007 (Microsoft, Redmond, WA, USA) and JMP 3.2 statistical software (SAS Institute, Cary, NC, USA). A $p$ value of $<0.05$ was considered statistically significant.

\section{RESULTS}

For the period 2.11.2008, 02:38 till 1.7.2014, 12:03, a total of 14985 EMS dispatches for dyspnea was identified; 4981 were classified as CPE, of these 4744 complete datasets were available and underwent further analysis. Table 1 shows baseline clinical characteristics of the patients. As much as $51.9 \%$ CPE episodes were associated with arterial hypertension, $5.9 \%$ with hypotension and $40.3 \%$ were accompanied by normotension $(\mathrm{p}<0.050)$. In 89 patients blood pressure values were not available.

Figure 1 shows the distribution of CPE episodes in separate intervals according to the intensity of the gravitational interaction. Occurrence of CPE episodes was highest in the periods with the weakest intensity of gravitational interaction (1.76 to $1.80 \mathrm{e} 1026 \mathrm{~N})$, that means when the Moon's distance from the Earth was highest, while in the period of the most intense gravitational interaction (2.26 to $2.30 \mathrm{e} 1026$ N) we observed the lowest proportion of CPE episodes (23.44 vs. $3.79 \%, \mathrm{p}<0.001$ ). Moreover, we observed two peaks of CPE incidence. After a steep decline in incidence with increasing gravity, we observed the next peak when gravitational interaction was $2.11-2.15 \mathrm{e} 1026 \mathrm{~N}$.

We identified different patterns of association of gravitational interaction and $\mathrm{CPE}$ occurrence in the groups of CPE patients with hypertension, normotension and hypotension (figure 2). While CPE patients with hypertension and normotension followed a two-peak profile, patients presenting with hypotension exhibited maximal incidence of CPE in the period with lowest intensity of gravitational interaction, but then followed a fluctuating profile of occurrence to a minimum in the period with the strongest gravity.

During the period of apogee, we observed significantly more CPE episodes than during perigee (672 versus 594, $\mathrm{p}=0.002$ ).

Breath rate, heart rate, presence of atrial fibrillation, diabetes mellitus, chronic pulmonary obstructive disease and peripheral oxygen saturation had no significant impact on CPE variation.

We did not observe any significant difference in the incidence of CPE episodes during the various lunar phases in the whole group or in the groups of CPE episodes with hypertension, normotension or hypotension (figure 3 ). 


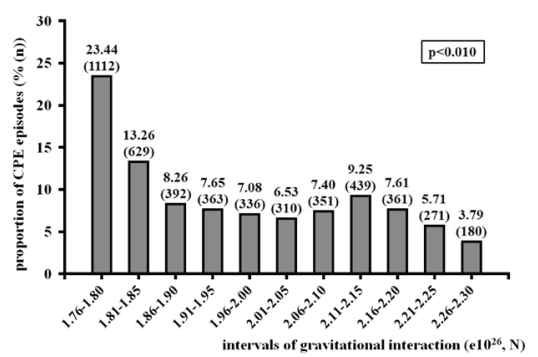

Figure 1. Association between the occurrence of $C P E$ episodes and the intensity of the gravitational interaction between the Earth and the Moon.

CPE, cardiogenic pulmonary edema.

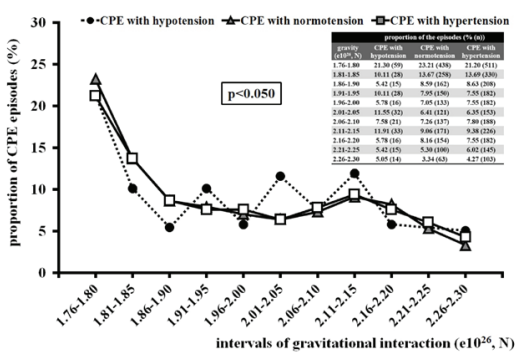

Figure 2. Association between the occurrence of $C P E$ episodes and the intensity of the gravitational interaction between the Earth and the Moon in the groups of CPE patients with hypertension, hypotension and normotension.

$C P E$, cardiogenic pulmonary edema. $p<0.05$ for the difference between CPE with hypotension and other groups

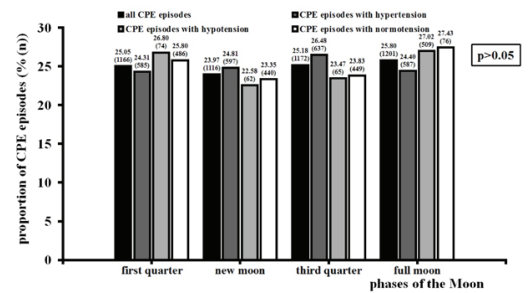

Figure 3. Proportion of CPE episode incidence during various phases of the moon. CPE, cardiogenic pulmonary edema.

Table 1. Baseline clinical characteristics of patients.

\begin{tabular}{|c|c|c|c|c|c|}
\hline VARIABLE & all CPE episodes & CPE with hypertension & CPE with normotension & CPE with hypotension & $\mathbf{p}$ \\
\hline Number of cases (n) & 4744 & 2463 & 1914 & 278 & \\
\hline age (years, mean $\pm S D$ ) & $75.0 \pm 10.5$ & $75.2 \pm 10.3$ & $74.5 \pm 10.7$ & $73.4 \pm 11.1$ & 0.006 \\
\hline men / women (\%) & $49 / 51$ & $49 / 51$ & $49 / 51$ & $47 / 53$ & $>0.050$ \\
\hline Diabetes mellitus (n / \%) & $1424 / 30.0$ & $726 / 29.5$ & $615 / 32.1$ & $83 / 29.5$ & $>0.050$ \\
\hline COPD / asthma (n / \%) & $513 / 10.8$ & $263 / 10.7$ & $219 / 11.4$ & $31 / 11.0$ & $>0.050$ \\
\hline \multicolumn{6}{|l|}{ initial heart rhythm } \\
\hline sinus rhythm (n / \%) & $2668 / 56.2$ & $1379 / 56.0$ & $1131 / 59.1$ & $158 / 56.2$ & $>0.050$ \\
\hline atrial fibrillation (n / \%) & $1239 / 26.1$ & $643 / 26.1$ & $522 / 27.3$ & $74 / 26.3$ & $>0.050$ \\
\hline $\begin{array}{l}\text { other rhythm / not known } \\
(\mathrm{n} / \%)\end{array}$ & $840 / 17.7$ & $441 / 17.9$ & $350 / 18.3$ & $49 / 17.5$ & $>0.050$ \\
\hline $\begin{array}{l}\text { number of STEMI patients } \\
(\mathrm{n} / \%)\end{array}$ & $222 / 4.7$ & $74 / 3.0$ & $97 / 5.1$ & $51 / 18.3$ & $<0.001$ \\
\hline $\begin{array}{l}\text { systolic arterial blood pressure } \\
(\mathrm{mm} \mathrm{Hg}, \text { mean } \pm \mathrm{SD})\end{array}$ & $149 \pm 39$ & $178 \pm 25$ & $124 \pm 14$ & $81 \pm 9$ & $<0.001$ \\
\hline $\begin{array}{l}\text { diastolic arterial blood pressure } \\
(\mathrm{mm} \mathrm{Hg}, \text { mean } \pm \mathrm{SD})\end{array}$ & $84 \pm 22$ & $97 \pm 16$ & $74 \pm 12$ & $46 \pm 19$ & $<0.001$ \\
\hline $\begin{array}{l}\text { mean arterial blood pressure } \\
(\mathrm{mm} \mathrm{Hg}, \text { mean } \pm \mathrm{SD})\end{array}$ & $105 \pm 26$ & $124 \pm 17$ & $91 \pm 12$ & $57 \pm 12$ & $<0.001$ \\
\hline $\begin{array}{l}\text { heart rate (beats/min., } \\
\text { mean } \pm \text { SD) }\end{array}$ & $99 \pm 26$ & $101 \pm 24$ & $97 \pm 25$ & $97 \pm 31$ & 0.010 \\
\hline SpO2 $(\%$, mean \pm SD) & $85 \pm 13$ & $85 \pm 11$ & $87 \pm 12$ & $80 \pm 17$ & $<0.001$ \\
\hline breath rate (breaths/min., & $24 \pm 6$ & $24 \pm 6$ & $22 \pm 7$ & $23 \pm 7$ & 0.015 \\
\hline
\end{tabular}

mean \pm SD)

$\mathrm{p}$ value was calculated for the differences between the subgroups of CPE with hypertension, normotension and hypotension COPD, Chronic Obstructive Pulmonary Disease; CPE, cardiogenic pulmonary edema; SpO2, peripheral capillary oxygen saturation; STEMI, ST-elevation myocardial infarction.

\section{DISCUSSION}

The main finding of our study is that, in a large number of cases, we identified a significant association between the intensity of gravitational interaction between the Earth and the Moon and occurrence of cardiogenic pulmonary edema treated by EMS. The pattern of association was found different for CPE with hypertension and hypotension. On the other hand, we did not find any effect of the lunar phases during the synodic lunar cycle on the incidence of CPE.

Variation of biological processes in time is a natural characteristic of living organisms. These events oscillate under the influence of exogenous environmental oscillators 
which are monitored by an endogenous system comprising peripheral and central biological clocks. (14) Among the main sources of exogenous oscillations are the Earth's rotation around its axis, rotation of the Moon around the Earth and the Earth's rotation around the sun. Interaction of the Earth and the Moon is expressed by a significant influence on the hydrosphere, atmosphere and Earth's crust in the form of tidal force. A reliable lunar cycle is also seen in living organisms, especially in marine animals. (15) It is not a surprise that the occurrence of cardiovascular emergencies such as stroke, acute aortic syndrome, acute coronary syndromes, sudden cardiac death, pulmonary embolism and cardiogenic pulmonary edema have been shown to be closely related to circadian, circaseptan and circaunnal variation. (16-20) The potential impact of the Moon and the Earth's interaction on human biorhythms may be mediated by the periodic fluctuations of night glow between a new moon and full moon. However, it has been repeatedly demonstrated that cyclic alternation of lunar phases has no significant effect on the occurrence of cardiovascular emergencies. $(1,2,9,10)$ This observation was confirmed also in our survey.

Another influence on human biorhythms can be due to regular variation of intensity of gravitational interaction on the basis of fluctuations in the distance between the Earth and the Moon. This happens regularly due to orbital excentricity of the Moon, with the distance between the Moon and Earth varying from around $356334 \mathrm{~km}$ to $405503 \mathrm{~km}$. (21) Wake et al. published an investigation of the effect of Moon gravity on the occurrence of acute myocardial infarction at 5 hospitals. They found, in 1369 consecutive patients, that there was an increase in incidence when the distance between the centre of the Moon and the Earth was more than $399864 \mathrm{~km}$, or in other words, when the gravity of the Moon was the least intensive. (12) An identical association was found in a retrospective cohort analysis of 1007 consecutive births performed by the same research group. (8) There are two major limitations in both studies. The authors performed in-patient cohort analysis and it may not represent all cases in the geographic region. Second, the authors did not use an appropriate formula for gravity calculation. They included in it only the weight of the Moon. However, the formula described the gravitational interaction between two celestial bodies and therefore, it is necessary to consider the product of weight of both the Moon and the Earth, which results in a force higher by 15 orders. Nevertheless, in principle, Wake et al. found a similar profile to the occurrence of myocardial infarction and number of births, as was the major finding in our study, i.e., that the occurrence of CPE episodes significantly increases during weaker gravitation periods and vice versa. We want to emphasize that we are not able to explain the described phenomena in more detail. It is likely that there is no direct causal relationship but it is rather a series of more intermediary events directly or indirectly affecting the final biological phenomenon. Anyhow, we assume that it is not a random association. Similar results were observed in two very distant geographical regions, and in our study, more than 4000 episodes of CPE were enrolled, which we consider a sufficiently large sample to be reliable. Interestingly, we identified different patterns of CPE occurrence variation in different clinical types of CPE. This should be the subject of further research.

Research on the phenomena affecting rhythmic fluctuation of the occurrence of cardiovascular emergencies is of major importance for several reasons. First, it allows a deeper understanding of its pathophysiology with respect to chronobiology. Second, monitoring variability of occurrence of emergencies in the field may help optimize operation of emergency medical systems, particularly in the allocation of occupancy of day and night shifts and in preparedness for the increased workload. Third, it may be of great importance in the secondary prevention of emergencies, which incidence is subject to regular occurrence of variability in time. CPE is one of the candidate diseases for such a chronotherapeutic approach. Pronounced circadian variation has been demonstrated for CPE occurrence and a casual relationship with hypertension, particularly with nondipper/riser profile and with the presence of excessive morning surge. $(22,23)$ Moreover, our results indicate that the chronobiological approach may be considered even beyond the horizon of the twenty four hour cycle. Although the causal relationship between gravitational interaction between the Earth and the Moon and the occurrence of CPE has not been detected so far, it is easy to automatically calculate the periods with increased risk of $\mathrm{CPE}$ occurrence in advance. Especially in patients with congestive heart failure and in those patients who have already suffered from a CPE episode, it can be considered wise to concentrate maximum preventive effort in terms of avoiding excessive physical activity, reduced salt intake, strict infection prevention and careful home blood pressure monitoring on these days, especially in unstable and decompensated patients.

\section{STUDY LIMITATIONS}

The major limitation of the presented study is that it is a retrospective and not prospective survey with all general limitations. Another limitation lies in the selection of the patients with CPE. It was based on the prehospital clinical assessment. However, careful retrospective stepwise re-evaluation of each event was performed to exclude all cases in which the cause of shortness of breath was other than CPE or the cause was unclear, to maximize homogeneity of selected patients. The patients who died of CPE before contacting EMS were not included. However, the latter is the common limitation of epidemiological studies. Finally, the data were collected from one central European region and the results should not automatically be applied to other geographic and socio-economic regions.

\section{CONCLUSIONS}

We conclude that a significant association of intensity of gravitational interaction between the Earth and the Moon and occurrence of cardiogenic pulmonary edema treated by EMS has been identified in a large cohort of cases in our study. A prospective approach in future research is warranted. Recognition and understanding of chronobiological features of cardiovascular emergencies may optimize emergency medical service operation, and imbedding a chronotherapeutic pattern into secondary prevention measures may improve long term management of these diseases. 


\section{REFERENCES}

1. Kanth R. Impact of lunar phase on the incidence of cardiac events. World J Cardiovasc Dis 2012;02:124-8.

2. Wende R, von Klot S, Kirchberger I, Kuch B, von Scheidt W, Peters A, et al. The influence of lunar phases on the occurrence of myocardial infarction: fact or myth? The MONICA/KORA Myocardial Infarction Registry. Eur J Prev Cardiol 2013;20:268-74.

3. Shuhaiber JH, Fava JL, Shin T, Dobrilovic N, Ehsan A, Bert A, et al. The influence of seasons and lunar cycle on hospital outcomes following ascending aortic dissection repair. Interact Cardiovasc Thorac Surg 2013;17:818-22.

4. Kuehnl A, Herzog M, Schmidt M, Hornung H-M, Jauch K-W, Hatz RA, et al. The dark side of the moon: impact of moon phases on long-term survival, mortality and morbidity of surgery for lung cancer. Eur J Med Res 2009;14:178-81.

5. Snoyman P, Holdstock TL. The influence of the sun, moon, climate and economic conditions on crisis incidence. J Clin Psychol 1980;36:884-93.

6. Pokorny AD. Moon phases and mental hospital admissions. J Psychiatr Nurs Ment Health Serv 1968;6:325-7.

7. Rovensky J, Mikulecky M. Pseudogout: Timing of attacks and the cosmogeophysical environment. Clin Exp Rheumatol 2000;18:5079.

8. Wake R, Misugi T, Shimada K, Yoshiyama M. The effect of the gravitation of the moon on frequency of births. Environ Health Insights 2010;4:65-9.

9. Alves DW, Allegra JR, Cochrane DG, Cable G. Effect of lunar cycle on temporal variation in cardiopulmonary arrest in seven emergency departments during 11 years. Eur J Emerg Med 2003;10:225-8.

10. Eisenburger P, Schreiber W, Vergeiner G, Sterz F, Holzer M, Herkner H, et al. Lunar phases are not related to the occurrence of acute myocardial infarction and sudden cardiac death. Resuscitation 2003;56:187-9.

11. Nadeem R, Nadeem a, Madbouly E, Molnar J, Morrison J. Effect of a full moon on mortality of patients admitted to the ICU. Crit Care 2012;16:P499.

12. Wake R, Yoshikawa J, Haze K, Otani S, Yoshimura T, Toda I, et al. The gravitation of the moon plays pivotal roles in the occurrence of the acute myocardial infarction. Environ Health Insights 2008;1:63-6.

13. Mancia G, Fagard R, Narkiewicz K, Redon J, Zanchetti A, Böhm M, et al. 2013 ESH/ESC guidelines for the management of arterial hypertension: The Task Force for the management of arterial hypertension of the European Society of Hypertension (ESH) and of the European Society of Cardiology (ESC). Eur Heart J 2013;34:2159-219.

14. Bass J. Circadian topology of metabolism. Nature 2012;491:348-56.

15. Sivaprasad, S. Circadian Biorhythms - Present Understanding and Status of Research. Indian J Appl Res 2014;IV:729-32.

16. Buff DD, Calikyan R, Neches RB, Bavli SZ. Circadian patterns in the onset of cardiogenic acute pulmonary edema. Clin Cardiol 1997;20:261-4

17. Cohen MC, Rohtla KM, Lavery CE, Muller JE, Mittleman M a. Meta-analysis of the morning excess of acute myocardial infarction and sudden cardiac death. Am J Cardiol 1997;79:1512-6.

18. Elliott WJ. Circadian variation in the timing of stroke onset: a meta-analysis. Stroke 1998;29:992-6.

19. Hakim H, Samadikhah J, Alizadehasl A, Azarfarin R. Chronobiological rhythms in onset of massive pulmonary embolism in Iranian population. Middle East J Anaesthesiol 2009;20:369-75.

20. Vitale J, Manfredini R, Gallerani M, Mumoli N, Eagle KA, Ageno W, et al. Chronobiology of acute aortic rupture or dissection: a systematic review and a meta-analysis of the literature. Chronobiol Int 2015;32:385-94.

21. Mcgraw-Hill. Encyclopedia of Science and Technology. 7th ed. McGraw-Hill; 1992.

22. Mallion J-M, Neuder Y, Ormezzano O, Rochette GB, Salvat M, Baguet JP. Study of nycthemeral variations in blood pressure in patients with heart failure. Blood Press Monit 2008;13:163-5.

23. Shin J, Kline S, Moore M, Gong Y, Bhanderi V, Schmalfuss CM, et al. Association of diurnal blood pressure pattern with risk of hospitalization or death in men with heart failure. J Card Fail 2007;13:656-62. 